\title{
Mental Disorder Recognition Using Face Based Image Retrieval Technique In The Compressed Domain \\ Irianto. Suhendro ${ }^{1}$,Sri Lestari ${ }^{2}$, and Fitria $^{3}$ \\ Department of Informatics, Darmajaya Informatics and Business Institute ${ }^{1}$ \\ suhendroirianto@ymail.com \\ Department of Informatics, Darmajaya Informatics and Business Institute ${ }^{2}$ \\ srilestari@ymail.com \\ Department of Information System, Darmajaya Informatics and Business Institute ${ }^{3}$ \\ fitria@ymail.com
}

\section{ABSTRACT}

\begin{abstract}
In this paper we explore content based image retrieval to identify mental disorder based on face expression the compressed domain. The work was conducted by constructing an application or tool which automatically detect and recognize someone experienced mental disorder based on face recognition. In this research we used more than 50,000 face images which consist of normal face expression with smile, sad, laugh, and angry expression. We wrote two face detection and face recognition algorithms to decide whether someone suffered or not. This work was carried out to built an automatic tool of face detection and recognition very fast and accurately. Face detection done to separate between face area and non face area in order focus on face recognition. In this work we used face image with normal pose without obstacles and facing toward with 90 degree. In the experiments we applied more than 50 queries from each category of total of two categories mental disorder. Our algorithms demonstrate good effectives of image retrieval in term of Precision and recall.
\end{abstract}

\section{Indexing terms/Keywords}

Recognition; matching; retrieval; face.

\section{Academic Discipline and Sub-Disciplines}

Informatics, image retrieval

\section{SUBJECT CLASSIFICATION}

Computer Sciences

\section{TYPE (METHOD/APPROACH)}

Experimental Research

\section{Council for Innovative Research}

Peer Review Research Publishing System

\section{Journal: INTERNATIONAL JOURNAL OF COMPUTERS \& TECHNOLOGY}

Vol 12, No.1

editor@cirworld.com

www.cirworld.com, member.cirworld.com 


\section{INTRODUCTION}

Face recognition and face exspression detection are which area interest of many researchers in the area of image analysis, pattern recognition, and biometrics. $[1,4,6,7]$. Face recognition is a complex pattern recognition, it is considered as multi dimentional classification process and signal matching. Face recognition can be clasificied into two categories feature based and template based. Feature based known as geometric based whilst template based known as photometric based [13]. This work applied to face face detection from the more than 50,000 face images. The system can be used to detect whether one's expericed of mental disorder not. We applied several face images with smile, angry, sad, scary, and laugh face expressions.

In this works normal faces are used with fordward pose 90 degree with and without obstacles. We also used JPEG images since JPEG has many benefits as such as reduce storage as well as fast data transfer. It also simplify algorithm calculation in face detection and face recognition. The objectives of this work is to recognize whether one has experienced mental disorder or not, In order to do that the system generates face exression pattern. Determining one's suffered or not can be known by comparing trainning image to images in the database. The comparation carried out based on i). detection of highest value of radial and regular symetric of images, ii). Location of eyes and mouth, and iii). Detection of mid of face area.

\section{REL ATED WORKS}

\section{Content Based Image Retrieval and JPEG.}

Content-based image retrieval (CBIR) is a low-level based features based retrieval simply based on the content or existing image [1]. Some content-based image retrieval have been done up to this time, including: Blobworld [2], the system PicToSeek [3], C-BIRD [4], and MARS system [5]. Meanwhile International standard compression has now been widely introduced and is known as JPEG and MPEG. JPEG image has been very popular as a ISO / ITU-T standard and is patented in the 1990s, some models have been defined by the JPEG [6] including the baseline model, lossless, progressive and hierarchical. Algorithm of digital image compression can be explained as follows [6].

- Original pixel divided into blocks with $8 \times 8$ dimensions, which amounts to 64 pixels where each pixel value is shifted from unsigned integers in the range $\left[0,2^{p}-1\right]$ to a signed integer in the range $\left[-2^{p}-1,2^{p}-1\right]$

- Then every pixel within the block $(\mathrm{Bi})$ are processed through the 2D Discrete Cosine Transform function and produce DCT blocks $\left(\mathrm{Bi}^{*}\right)$, and every single $\mathrm{DC}$ coefficient (which is the average intensity of all the blocks) and AC coefficients of the number 63 on each block, can be formulated as follows:

$$
\mathrm{F}(\mathrm{u}, \mathrm{v})=\frac{1}{4} \sum_{\mathrm{x}=0 \mathrm{y}=0}^{7} \sum_{\mathrm{y}}^{7} \mathrm{C}(\mathrm{u}) \mathrm{C}(\mathrm{v}) \mathrm{f}(\mathrm{x}, \mathrm{y})\left[\cos \left(\frac{(2 \mathrm{x}+1) \mathrm{u} \pi}{16}\right) \cos \left(\frac{(2 \mathrm{y}+1) \mathrm{v} \pi}{16}\right)\right]
$$

where: $u, v$ vary in accordance with the direction of columns and rows, $C(u), C(v)=$ for $u, v=0$.

- After that quantizes done on a 64 coefficient is by using the following equation:

$$
F Q=\text { int } \operatorname{Round} \frac{F(u, v)}{Q(u, v)}
$$

- Implementation of a zig-zag order and then the coefficient of each block in quantizes Application of entropy code on each coefficient either with Huffman or arithmetic.

\section{Image Searching.}

Traditional image searching and retrieval were carried out based on query-by-example (QBE) starting from the input image into the system (query image) are compared to the existing image in the database, several studies have been made by researchers, among them [8]. In face image based retrieval, they stated that standard query image can be a mental image. Furthermore they said that their research focus on face features, moreover all the algorithms developed can be applied to other domains, for example in clothing, home furnishings, and paintings, and mental face images. One example of effective mental image searching has been stated by Cox [9].

Image searching in the compressed domain by using many methods have been done previously and provide satisfactory and effective results compare to image searching in the pixel domain [10]. As has been proven that with a certain level of compression, then search the face to give even better results than a search on the DCT domain.

\section{Face recognition}

Although many automatic face recognition techniques are successfully applied in many areas of daily life, but the task of face recognition based only on the scale bias. Research topic in face recognition is still a challenge, especially in the uncontrolled environment [11]. Meanwhile, to match with face image that is in the database or gallery we use the Euclidean distance calculation, if Euclidean distance equal to zero then the image will be exactly the same query image is in the database. 
Face recognition can be done by using the chromatic color components, Hue and Saturation as were done by Zhao in Petrou [8]. Face can be considered as unique features of man, even twins their faces still different although they are very similar. Human expertise to recgnize a face can be done easily even if face's appearances influenced by its expression, age, and obstacles (glasess, hat, hijab).

Since face is the window or form part of the body that describes the emotions and circumstances of our lives. Face can be considered as unique features of human, even twins they are will remain distinct even though his face looks exactly the same. Human expertise to recognize faces can be done easily influenced by even the appearance of face expression, age, and obstructions such as glasses or hairstyle changes. However, to detect faces with appropriate especially for faces in a completely new is not easy and there are many issues relating to the above, these problems include: detection of a model's face, recognition, analysis face expressions, and classification based on physical features [7] .

\section{RESEARCH METHODS}

\section{The Ground Truth}

Firstly, this work was done by creating a database of face images with JPEG format, so that the image of the face, which mostly non-JPEG format must first be converted into JPEG format. Face image database constructed in the range of between 50,000 images and is composed of several normal face image (face looking straight ahead), the image database is also equipped with face images from mental disorder hospital. The three categories face database constructed based on Seager [13] works namely: i). when eyebrows move to the middle of forehead and eye move to top right, ii). lips move hesitant and iii) when ones touch nose and mouth and close his mouth. Meanwhile, the system generated in this work done by using Visual NET platform. The output is expected to deliver a method which to provide a significant contribution to recognize mental disorder experienced.

The reason of in this study using JPEG images since JPEG images have several advantages compared to other formats, some of its advantages are: (i). file size is relatively small compared to other formats (image can be DC up to reach $70 \%$ without reducing the quality of the original image), (ii). Easy and simple algorithm due to the image is divided into blocks of DCT coefficients, (iii). Most cameras today have used the JPEG format. Moreover, JPEG is an international standard format for images on the Internet and in multimedia devices today.

$\mathrm{YCbCr}$ color space Component is used on the grounds because $\mathrm{Y}$ is a component that represents information luminance and $\mathrm{CbCr}$ chrominance represent information used in the JPEG format so that it will reduce complexity. Moreover, compared with the color RGB, YCbCr more in line with the human visual system. Texture extraction is done by selecting three DCT coefficients, namely: DC coefficient representing the energy information, the AC coefficients which represent frequency information, and the DCT coefficients which represent the direction or trend information about the image.

Analysis of the perpendicular face of the face or profile of the face is often effective without the participation or any previous knowledge. Meanwhile, for face image retrieval algorithm is used which can be explained as follows:

For example face image query, $q$, and image $n$ the database $X$, all face images extracted, ..,, $q 1 q 2$, and $X_{i} X_{j}, \ldots$. each face space.

- $\quad$ Then calculate the euclidean distance $d\left(q_{i}, x_{j}\right)$ for all pairs $(q i, x j)$

then

$\bullet$

$$
d_{\text {wajah }}(q, X)=\min i, j\left\{d\left(q_{i}, x_{j}\right)\right\} \text { where } i=1, \ldots \ldots, i, j=1, \ldots, j
$$

\section{Research steps}

Research is conducted in two phases; first phase involves the collection of face image of more than 50,000 face images. In this first phase, activities will be focused on activities and face features extraction. Fig.1, describes the flow of activities which constitute the face extraction. 


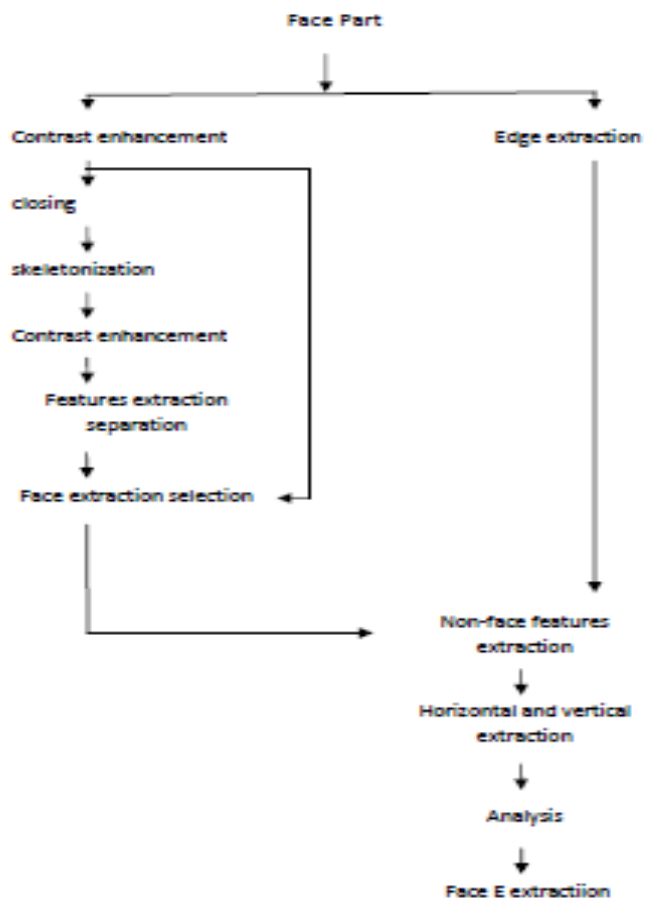

Figure 1. Face features extraction

To stage face recognition, this works use to implement image retrieval by image query into the system, which a imple implementation of image retrieval based on face detection feature. Database used in this study is a combination or mixture of some face expressions such as face was mediocre, angry face, smiling, shouting, and laughing, while also used illumination (some variation of radiation from the left side, right side, and radiation from both sides).
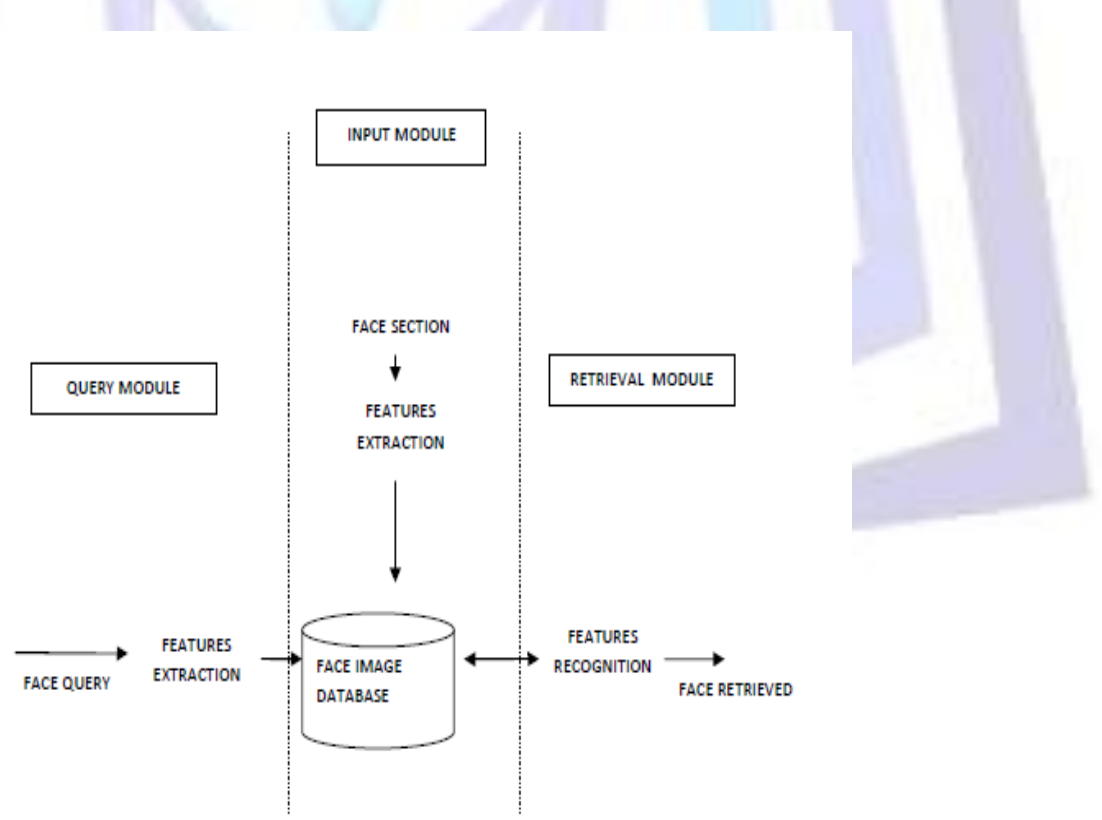

Figure 2. Diagram of face recognition retrieval system

The database used in this study is a combination or mixture of some face expressions such as face was mediocre, angry face, smiling, shouting, and laughing, while also used illumination (some variation of radiation from the left side, right side, and radiation from both sides). 


\section{Evaluation of Effectiveness of Searching}

To measure the similarity or degree of similarity between the query face image (the reference face) with face image is in the database, then used the Euclidean distance formula which can be defined as follows:

$$
\mathrm{d}(\mathrm{Q}, \mathrm{I})=\frac{\sum_{i=0}^{M}\left(Q_{i}-I_{i}\right)^{2}}{M}
$$

Where $Q$ is the query face image and I is a vector of image features that exist in the database, while $M$ represents the number of elements present in the image features. As for calculating effectiveness (effectives) of face images used search precision and recall parameters that can be written as follows:

$$
\begin{aligned}
& \text { Precision }=\frac{\sum \text { relevant images retrieved }}{\sum \text { images retrieved }} \\
& \text { Recall }=\frac{\sum \text { relevant image retrieved }}{\sum \text { relevant image in category in the database }}
\end{aligned}
$$

The greater the value of precision and recall, the more effective methods or techniques used by search, Precision has a maximum value 1 and minimum 0 .

\section{Faces Searching Algorithm}

The algorithm of the method applied in this study is to explore the features available in the DCT coefficients. Each DCT coefficient is a vector containing the energy to build a histogram in the process of matching during the search process and the introduction of face images from the database. Sequence or algorithm of this technique can be explained as follows:

1. Input face image

2. Convert RGB Image into YCbCr and HVS component, get face candidate

a. Generate Key Indexing from DCT coefficient of 2D matrix image calculate I(query Image) as follow:

$$
H(i)=\{h 0, h 1, \ldots, h 63) \text {, and } h i=\sum_{i=0}^{63} Q(u, v)
$$

$Q(u, v)$ is DCT coefficient at row $u$, and column $v$

Similar step 3 for image in the database.

$H(i)=\{h 0, h 1, h 2, \ldots, h 63\}$, and $h i=\sum_{i=0}^{63} C(u, v)$

$d(u, v)$ is DCT coefficient at row $u$ and column $v$.

3. Calculate similarity between image query and image in the database using this formula:

$$
D(I q, I d) M \text { in }=\frac{\sqrt{\sum_{i=0}^{N}\left(I_{q i}-I_{d i}\right) .\left(I_{q i}-I_{d i}\right)}}{N}
$$

Where $D$ is distance between Iq vector Id vector (face in the database). Whilst $N$ is number of block of related face image. $D$ value has range 0 and 1 , if $D=0$ then image query is exactly the sane to image retrieved from the database.

4. Range face image retrieved based $D$ value calculated as ascending order.

For Number_of_block $=1$ to $N$

For $u=0$ to 63

For $v=0$ to 63

End

$D((\operatorname{lq}(u, v), \operatorname{ld}(u, v)))$;

End

End

5. Display 20 images similar retrieved

6. For next image query, repeat steps 1 to 6 . 


\section{RESULTS AND DISCUSSION}

\section{Ground Truth}

In this research we use a database which consist of no less than 50,000 face images. The images consist of face with normal pose, smile, laugh, sad, happy, stress ,and face with mental disorder expression. Face with normal pose means straight forward 90 degrees without obstacles (no classes, no hat, and no wearing hijab).

\section{Effectiveness of Face With Mental Disorder Expression}

From 50 queries (reference face) was deployed as test taken from no less than 500 training images (training face images) in this work, showing that the effectiveness is quite good because most of the Precision gained greater than 0.67 or $67 \%$, more detail can be examined from table 1 .

\section{Query Modul Query Wajah and Matching Algorithm}

Even though the application we made from this work not ready as a commercial software yet, its algorithm is quite good to detect and recognize he / she whom suffered mental disorder based on his or her face expression. The algorithm of this application can be describes as follow:

1. Face image query suspected mental disorder

a. Face image query

b. Convert RGB image to $\mathrm{YCbCr}$ and HVS components

2. Extraction of statistical feature by calculating Eigenfaces value

3. Compute average of matrix $\Psi$, then subtracted from original image and stored into $\Phi i$ variable.

$$
\begin{aligned}
& \Psi=\frac{1}{M} \sum_{n-1}^{M} \Gamma n \\
& \Phi \mathbf{i}=\Gamma \mathbf{i}-\Psi
\end{aligned}
$$

4. Compute covariance matrix $C$ by using the following formula:

$$
\mathrm{C}=\frac{1}{\mathrm{M}} \sum_{n-1}^{M} \Phi_{n} \cdot \Phi_{n}^{T}
$$

5. Compute Eigenvector and eigenvalue from matrix covariance, where eigenvectors (eigenfaces) Ui and eigen values $\lambda i$ have been calculated.

6. Choose principal components of Face image

From eigenvectors $M$ (eigenfaces) ui, only the highest eigenvalue of $M^{\prime}$ chosen. The higher its eigenvalue, the more face characteristic features can be informed. Eigenfaces with lower eigenvalue can be eliminated. When eige face $M$ ' and Ui computed then training phase ends.

7. Compute similarity between image query and image in the database by using Euclidean Distance:

$$
\left.\mathrm{d}\left(\mathrm{x}_{\mathrm{i}}, \mathrm{x}_{\mathrm{j}}\right)=\sqrt{\sum_{\mathrm{r}=1}^{\mathrm{n}}(\mathrm{ar}}-(x i)-\operatorname{ar}(x j)\right)^{2}
$$

8. Sort retrieved images based on Euclidean Distance values as ascending order For Number_of_block $=1$ to $\mathrm{N}$

$$
\begin{aligned}
& \text { For } u=0 \text { to } 63 \\
& \text { For } v=0 \text { to } 63 \\
& D((l q(u, v), l d(u, v))) \text {; } \\
& \text { End } \\
& \text { End }
\end{aligned}
$$

End

9. Display the most similat 20 images retrieved.

10. For next quer repeat step 1 to 8.

\section{Face detection Algorithms}

In this work algorithm used to explore DCT coefficients features which the coefficients is considered as vector. The vector consists of energy to build a hsitogram in matching process of face recogniton into image database. Face detection can be examined from this following algorithm.

Read image

For $i=0$ to img_height

For $j=0$ to img_width

Convert image to $\mathrm{YCrCb}$

For $i=0$ to img_height 
For $j=0$ to img_width

Convert image to Binary image

For $i=0$ to img_height

For $j=0$ to img_width

$Y=i m g Y U V(I, j), Y$

$U=i m g Y U V(I, J), U$

If $U>150$ and $U<200$ then $V=255$

Else $U=0$

$V=i m g Y U V(I, J) V$

$f V<=140$ and $V>=170$ then $V=255$

else $V=0$

\section{Edge detection}

For $Y Y Y=1$ to Pic Y.height-1

For $X X X=1$ to PicX.width-1

End

Display Detected image

Table 1. Face matching effectiveness

\begin{tabular}{|c|c|c|c|c|c|c|c|c|}
\hline QUERIES & PRECISION & RECALL & QUERIES & PRECISION & RECALL & QUERIES & PRECISION & RECALL \\
\hline 1 & 0,61 & 0,20 & 18 & 0,81 & 0,10 & 35 & 1,00 & 0,00 \\
\hline 2 & 0,61 & 0,40 & 19 & 0,79 & 0,30 & 36 & 0,98 & 0,35 \\
\hline 3 & 0,56 & 0,30 & 20 & 0,78 & 0,30 & 37 & 0,96 & 0,30 \\
\hline 4 & 0,55 & 0,25 & 21 & 0,77 & 0,45 & 38 & 0,95 & 0,35 \\
\hline 5 & 0,55 & 0,25 & 22 & 0,76 & 0,65 & 39 & 0,95 & 0,35 \\
\hline 6 & 0,50 & 0,25 & 23 & 0,75 & 0,05 & 40 & 0,95 & 0,10 \\
\hline 7 & 0,48 & 0,35 & 24 & 0,74 & 0,15 & 41 & 0,94 & 0,35 \\
\hline 8 & 0,47 & 0,35 & 25 & 0,73 & 0,10 & 42 & 0,90 & 0,10 \\
\hline 9 & 0,45 & 0,30 & 26 & 0,72 & 0,15 & 43 & 0,89 & 0,30 \\
\hline 10 & 0,44 & 0,35 & 27 & 0,65 & 0,25 & 44 & 0,88 & 0,30 \\
\hline 11 & 0,43 & 0,35 & 28 & 0,65 & 0,20 & 45 & 0,87 & 0,15 \\
\hline 12 & 0,35 & 0,45 & 29 & 0,64 & 0,25 & 46 & 0,86 & 0,35 \\
\hline 13 & 0,33 & 0,55 & 30 & 0,64 & 0,30 & 47 & 0,85 & 0,35 \\
\hline 14 & 0,30 & 0,40 & 31 & 0,63 & 0,25 & 48 & 0,84 & 0,35 \\
\hline 15 & 0,25 & 0,45 & 32 & 0,63 & 0,30 & 49 & 0,83 & 0,35 \\
\hline 16 & 0,20 & 0,45 & 33 & 0,62 & 0,25 & 50 & 0,82 & 0,10 \\
\hline 17 & 0,15 & 0,45 & 34 & 0,62 & 0,30 & & & \\
\hline
\end{tabular}

From Table 1 can be calculated that the average effectiveness of face matching in term of precision and recall are 0.67 and 0.28 , respectively, while graph in figure 1 shows precision and recall relations. Graph 3 explained, when precision value increase, oppositely the decrease of recall value. Rate of face images retrieval from the directory depends on the computer, the faster the processor, the faster indexing and retrieving. 


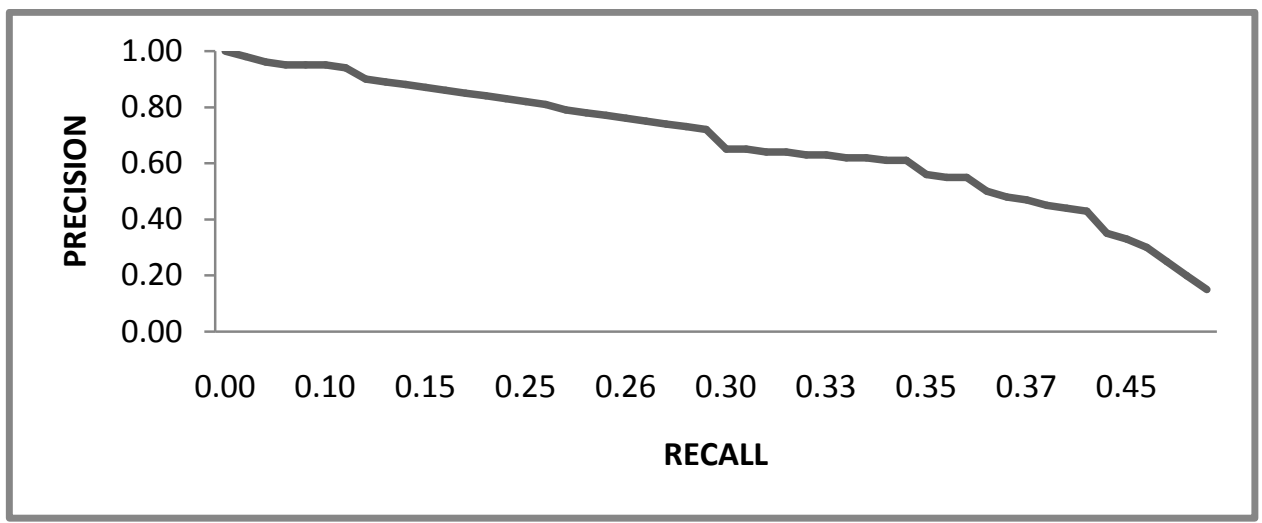

Figure 3. The effectiveness of mental disorder face recognition by precision and recall parameters

Similar to graph, graph 2 in fig.2 illustrated infectivity of mental face disorder recognition/retrieval of 50 queries. The graph displays that no zero value of precision since we choose mental disorder face from database as training image.

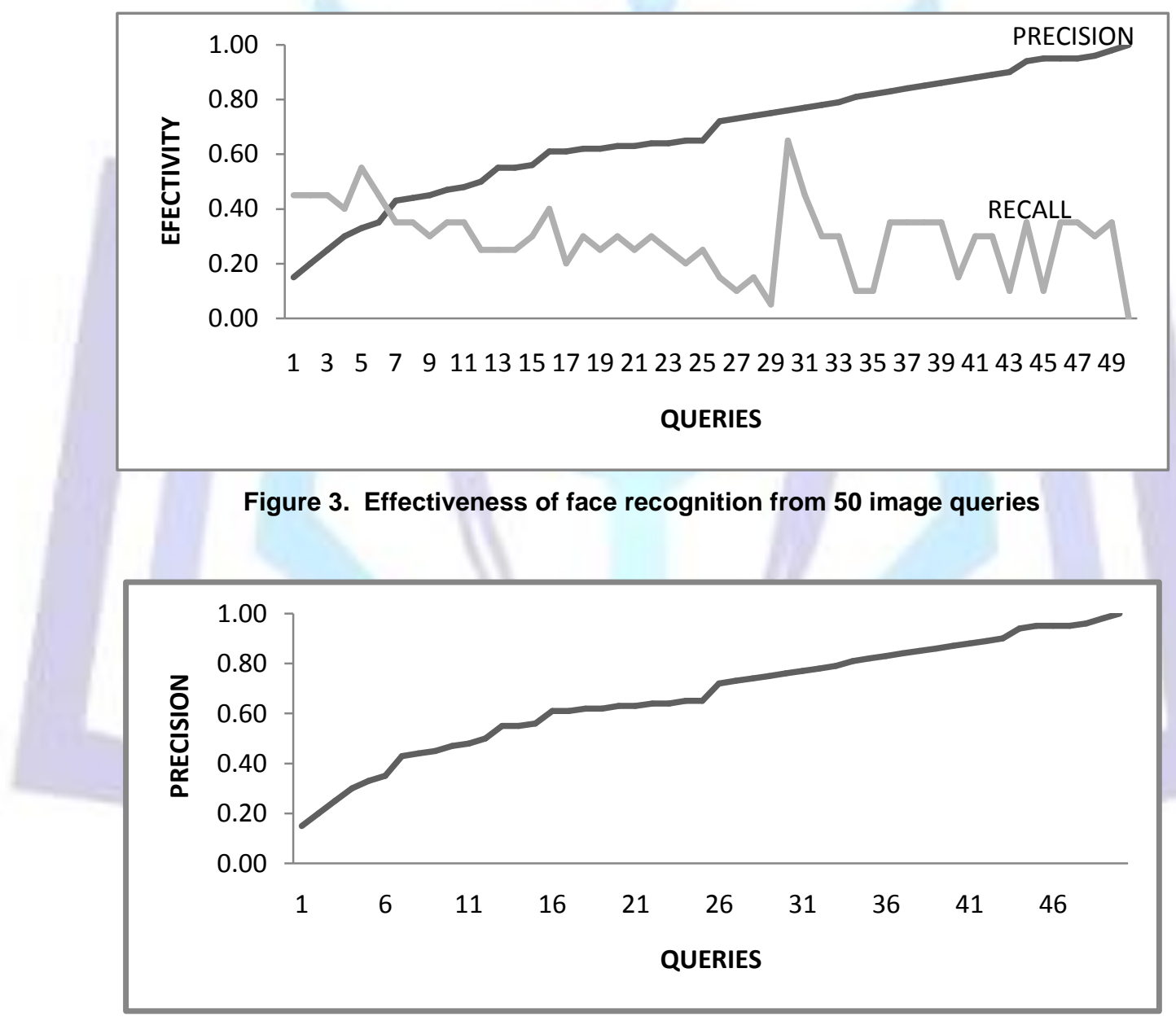

Figure 4. Precision of 50 image queries 


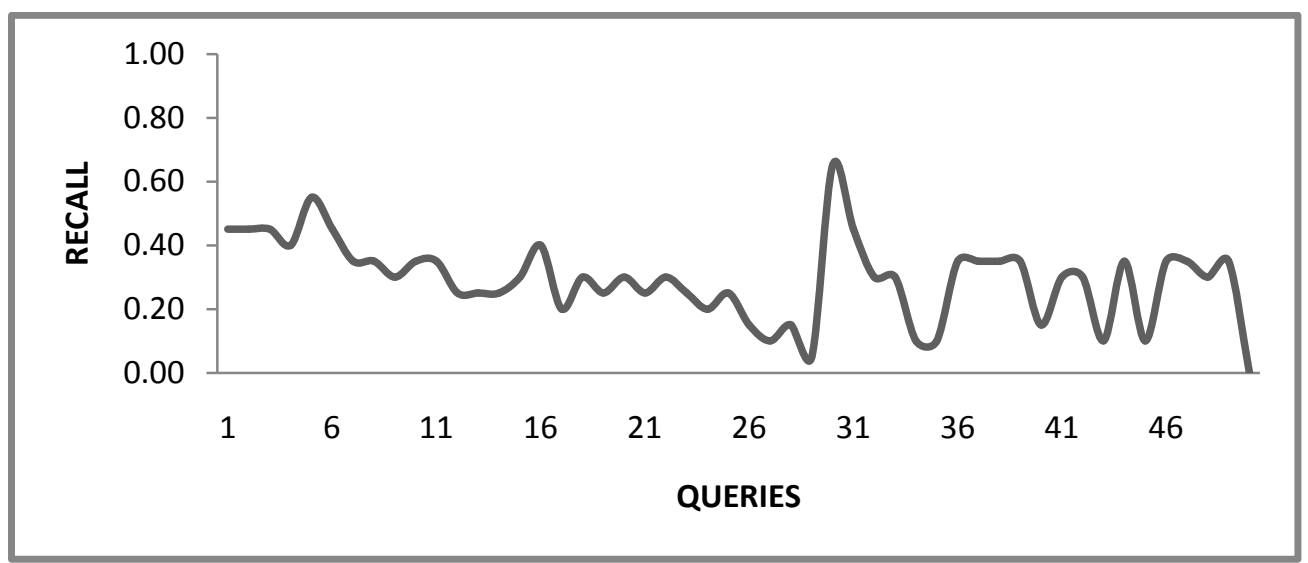

Figure 5. Precision of 50 image queries

\section{CONCLUSION AND FUTURE WORKS}

\section{Conclusion}

Several conclusions can be drawn from the results of the study face recognition that is the JPEG image file sizes are much smaller, does not reduce the information displayed. This is evidenced by the retrieval effectiveness of which is still quite good (about 0.55 ) by using the method of DCT feature extraction. In the algorithm used in the study, features - and not face face features are also applied to produce accurate results. Even though, GUI of the tool has not been considered ready as a commercial application, our algorithms show fair effectiveness in term of Precision and Recall.

\section{Future Works}

Existing and future research needs to be worked to improved the effectiveness of the algorithm by using larger database both in terms of both quantity and variety. We will try to apply other methods such as segmentation, neural network, and fuzzy logic for face detection and recognition.

\section{ACKNOWLEDGMENT}

We would like to thank to the Directorate General of Higher Education, Republic of Indonesia for supporting and funding with Hibah Bersaing fund. We also thank to the Research Department of Darmajaya Informatics and Business Institute for providing guiding and allowing us to use their laboratory to finish our work.

\section{REFERENCES}

[1]. Liang. K., C., and C.J. Kuo.2007. Wave Guide: A Joint Wavelet-Based Image Representation And Description System , IEEE Transaction on Image.Processing, Vol.8, 1999,pp. 1619-1629.

[2]. Carson. C, S. Belongie, H. Greenspan, dan J. Malik. 2007. Blobworld: Image Segmentation Using ExpectationMaximization and its Application to Image Querying, IEEE Trans. Pattern Analysis Machine Intelligent, Vol. 24, No. 8,pp. 1026-1038.

[3]. Gevers. T dan M. Arnold. 2000. PicToSeek: Combining Colours And Shape Invariant Features For Image Retrieval, IEEE Transaction on Image Processing, Vol.9, No.1, hal. 102-119.

[4]. Li. Z, Zaiane. R.O dan Yan. B. 1999.C-Bird: Content-Based Image Retrieval From Digital Libraries Using Illumination Invariance And Recognition Kernel, dalam: Wagner, R. (Ed.), Proceeding of The Ninth International Workshop on Database and Expert Systems Applications (DEXA '98), Vienna, Austria, August 2428, IEEE Computer Society, pp. 361-366.

[5]. Ramana et. al. 2012. A Prototype System using Lexical Chains for Web Images Retrieval Based on Text Description and Visual Features. International Journal on Computer Science and Engineering (IJCSE), Vol. 4No 05,pp.846-852.

[6] Wallace, Gregory K. The JPEG Still Picture Compression Standard, Commu nications of the ACM, vol. 34, no. 4, April 1991, pp. 30-44.

[7]. Petcharat Pattanasethanon and Charuay Savithi. 2012. Human Face Detection and Recognition using WebCam. Journal of Computer Science 8 (9): 1585-1593. 
[8]. Depeursinge et.al.2013. Benefits Of Texture Analysis Of Dual Energy Ct For Computer-Aided Pulmonary Embolism Detection. International Journal of Computer Assisted Radiology and Surgery. Journal no. 11548 .Pp.1-14. ISSN: 1861-6429.

[9]. Vassilios Stathopoulos and Joemon M. Jose.2012. Bayesian Probabilistic Models for Image Retrieval. JMLR: Workshop and Conference Proceedings $17,2^{\text {nd }}$ Workshop on Applications of Pattern Analysis,Pp.41-47

[10.] Irianto. Y.S And J. Jiang, DCT Based Content Descriptors For JPEG DC Image Indexing And Retrieval , Proceedings of EEngNet, 5th International Conference On E-Engineering \& Digital interprise Technology, $16^{\text {th }}$ $18^{\text {th }}$ August, 2006, China, Pp. 122-127. ISBN 2006243.

[11]. Adini, Y., Y. Moses, and S. Ullman. 1997. Face Recognition: The Problem Of Compensating For Changes In Illumination Direction. IEEE PAMI, 19(7), pp.721-732

[12]. Petrou, M., dan P. Bosdogianni. 2002. Image Processing: The Fundamentals. John Wiley \& Sons Ltd, England, 2005,Pp.. 265.

[13]. Sanders, E., Nathan, Emily. M, and Soderberg. 2013. Using Colors to Improve Photometric Mentality Estimates For Galaxies. Astrophysical Journal, Vol. 75, Issue 2.

[14]. Toure, M.L. and Z. Beiji, 2010. Intelligent Sensor For Image Control Point Of Eigenfaces For Face Recognition. Journal Computer Science, 6, pp.484-491.

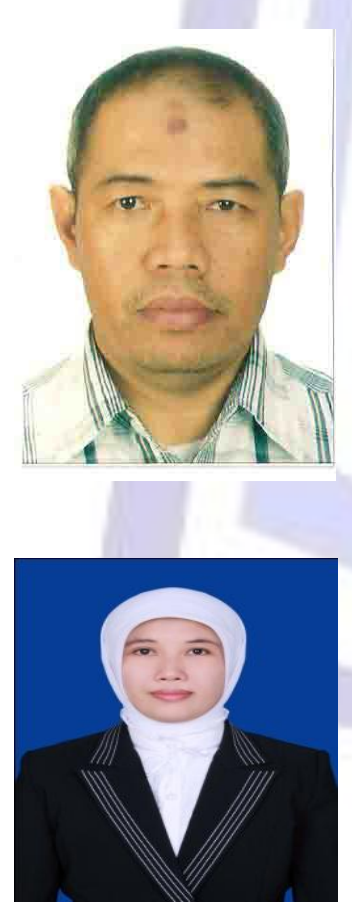

Suhendro Y. Irianto received his Master in computers science from The University of Indonesia, Jakarta Indonesia. He got Ph.D degree image Retrieval from the University of Bradford, United Kingdom in 2008. Currently he works as a researcher in Informatics Engineering department, Darmajaya Informatics and Business Institute, Indonesia. His interests are image retrieval, biometrics, pattern recognition, and multimedia database.

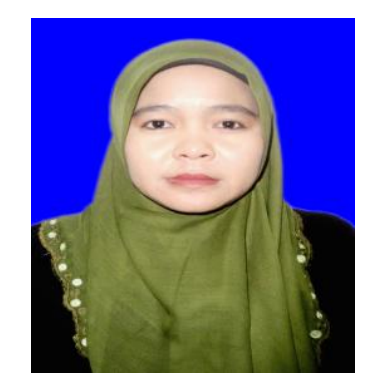

Sri Lestari received her Master in computers science from Gadjah Mada University, Yogyakarta, Indonesia. Currently she works as a researcher as well as a lecturer in Informatics Engineering department, Darmajaya Informatics and Business Institute, Indonesia. Her interests are artificial intelligent, case base reasoning, fuzzy logic, software engineering, and decision support system.

Fitria received her Master in computers science from Gadjah Mada University, Yogyakarta, Indonesia. Currently she works as senior a researcher in Informatics Engineering department, Darmajaya Informatics and Business Institute, Indonesia. Her interests are artificial intelligent, image processing, fuzzy logic, software engineering, and decision support system. 